\title{
Síndrome de burnout en docentes de una Unidad Educativa, Ecuador
}

\author{
Burnout syndrome in teachers of an Educational Unit, Ecuador
}

Síndrome de burnout em professores de uma Unidade Educacional, Equador

ARTÍCULO DE INVESTIGACIÓN

\author{
Angélica Manzano Díaz \\ angie_manzano7@hotmail.com \\ ORCID: 0000-0002-6891-7582 \\ Instituto Tecnológico Universitario Cordillera, Ecuador
}

Recibido 23 de noviembre 2020 | Arbitrado y aceptado 12 de diciembre 2020 | Publicado en diciembre 2020

\section{RESUMEN}

El síndrome de burnout es un conjunto de síntomas de desgaste emocional que afectan la salud $y$ el rendimiento laboral personalprofesional. El objetivo principal del estudio fue analizar el nivel de burnout que muestra el personal docente, midiendo la prevalencia de despersonalización, cansancio emocional y realización personal. El diseño de la investigación fue descriptivo, la técnica usada fue la encuesta, instrumento el cuestionario de Maslach Burnout Inventory (MBI) constituido por 22 ítems en forma de afirmaciones, sobre los sentimientos y actitudes del profesional en su trabajo y hacia los alumnos. Entre los principales hallazgos, el 29\% de los docentes de la unidad educativa padecen de cansancio emocional; la despersonalización fue del 17\%; y el $85 \%$ de los docentes presenta un alto nivel se realización. Se concluye que los docentes poseen una prevalencia baja del síndrome, existen docentes ubicados en la escala media y una población relativamente pequeña con una prevalencia alta que podría ser un factor de riesgo.

Palabras clave: Síndrome de burnout; docentes; agotamiento emocional; despersonalización; realización personal

\section{ABSTRACT}

Burnout syndrome is a set of symptoms of emotional exhaustion that affect health and personal-professional work performance. The main objective of the study was to analyze the level of burnout shown by the teaching staff, measuring the prevalence of depersonalization, emotional exhaustion and personal fulfillment. The research design was descriptive; the technique used was the survey, the Maslach Burnout Inventory (MBI) questionnaire instrument consisting of 22 items in the form of statements, about the feelings and attitudes of the professional in their work and towards the students. Among the main findings, $29 \%$ of the teachers in the educational unit suffer from emotional fatigue; depersonalization was 17\%; and $85 \%$ of teachers have a high level of achievement. It is concluded that teachers have a low prevalence of the syndrome, there are teachers located in the middle scale and a relatively small population with a high prevalence that could be a risk factor.

Key words: Burnout syndrome; teachers; emotional exhaustion; depersonalization; personal fulfillment 


\section{RESUMO}

A síndrome de burnout é um conjunto de sintomas de exaustão emocional que afetam a saúde e o desempenho pessoal-profissional no trabalho. 0 objetivo principal do estudo foi analisar o nível de burnout apresentado pelos docentes, medindo a prevalência de despersonalização, esgotamento emocional e realização pessoal. 0 desenho da pesquisa foi descritivo, a técnica utilizada foi a survey, instrumento questionário Maslach Burnout Inventory (MBI) composto por 22 itens na forma de afirmações, sobre os sentimentos e atitudes do profissional no seu trabalho e em relação aos alunos. Entre os principais achados, 29\% dos professores da unidade educacional sofrem de fadiga emocional; despersonalização foi de $17 \%$; e $85 \%$ dos professores têm um alto nível de desempenho. Conclui-se que os professores apresentam baixa prevalência da síndrome, existem professores situados em escala média e uma população relativamente pequena com uma alta prevalência que pode ser um fator de risco.

Palavras-chave: síndrome de burnout; professores; exaustão emocional; despersonalização; realização pessoal

\section{INTRODUCCIÓN}

El siglo XXI se ha caracterizado por un ritmo de vida cambiante $y$ acelerado, especialmente con un incremento pronunciado de las exigencias laborales. Esto responde a los requerimientos de la economía y demandas de la sociedad en general y es así como las personas insertas en este sistema laboral se ven obligadas a satisfacer dichas demandas adaptándose a las condiciones actuales. Por tanto, la profesión docente atraviesa por continuos cambios impulsados por las reformas en el sistema educativo por lo que, las expectativas y los retos que recaen en el profesorado incrementan cada vez más el nivel de exigencia, (Imbernón, 2017).

En las instituciones educativas cuando las condiciones laborales son deficientes, dado que en muchas ocasiones no brindan la seguridad y protección necesarias para los colaboradores, se convierten en una amenaza para la salud, provocando enfermedades laborales. Así mismo, en el quehacer docente, el hecho de no permitir que tengan autonomía y algún nivel de control sobre su trabajo, influye en el aumento del nivel de tensión psicológica del trabajador lo que genera el estrés laboral $\mathrm{y}$ otras enfermedades profesionales asociadas como un síndrome con características particulares, llamado burnout o síndrome de quemarse por el trabajo que se presenta mayormente en profesionales que prestan servicio directo a personas.

En el ámbito laboral, los profesionales más vulnerables a padecer el síndrome de burnout son aquellos que se caracterizan por presentar un buen desempeño laboral, comprometidos con su trabajo y que tienen expectativas altas sobre sus propias metas. Por lo que, cuando el resultado de su gestión no satisface las expectativas propias ni las de sus clientes, y además la organización no presta el apoyo necesario, estas personas se ven expuestas a un nivel alto de estrés y frustración.

Aunque existen varias definiciones del síndrome de burnout, Schaufeli (1998) lo definen como: "un estado mental persistente, negativo y relacionado con el trabajo, en individuos «normales», caracterizado principalmente por agotamiento, acompañado de estrés, sensación de competencia reducida, disminución de la motivación y el desarrollo de actitudes disfuncionales en el trabajo".

Aunque, teóricamente, existen tres dimensiones básicas del burnout (agotamiento, cinismo y baja eficacia personal), estudios empíricos abordados por Green, Walkley y Taylor (1991); Schaufeli (2005), Salanova (2000), Schaufeli, WB, Salanova, M., GonzálezRomá, V (2002), han demostrado que el agotamiento y el cinismo constituyen lo que se ha venido a denominar el «corazón del 
burnout". Por un lado, en el contexto educativo, el agotamiento hace referencia a la pérdida de recursos emocionales debido a las demandas de los estudiantes, los demás profesores y/o las propias familias de los estudiantes; por otro lado, el cinismo refleja indiferencia y actitudes distantes y cínicas hacia el trabajo que uno realiza (Cruz y Puentes, 2017)

En esta línea, los autores Moreno Jiménez, Garrosa-Hernández y González-Gutiérrez (2002) señalan que, cuando el profesor percibe una serie de fuentes de estrés es común que adopte actitudes de distanciamiento y despersonalizadas hacia los estudiantes entendiéndose esta conducta como un modo de afrontamiento al agotamiento experimentado. Además, el estudio muestra la importancia de determinadas variables sociodemográficas (edad, sexo, relación personal, número de hijos, nivel de enseñanza y situación laboral) en la explicación de los procesos de estrés y burnout.

En Ecuador, los docentes se encuentran en una situación compleja por el sistema de educación adoptado en los últimos años. Pombo (2015) en su obra Reforma educativa en Ecuador y su influencia en el clima organizacional de los centros escolares dice que, la reforma educativa se refiere a las decisiones que toma un Estado con el fin de orientar políticas educativas para superar las deficiencias detectadas en un sistema con resultados insatisfactorios en términos de calidad y eficiencia. Por lo que, toda reforma educativa surge de la evaluación del servicio educativo que brinda a sus ciudadanos y la influencia positiva o negativamente en la estructura social y educativa.

En el contexto socio-educativo el docente puede atravesar un estado de frustración y desmotivación debido a la falta de estrategias para reorientar los conflictos generados por la convivencia docente. Es así, como el burnout puede afectar a varios profesionales, pero en especial a aquellos que trabajan directamente con personas, como en el caso de los docentes. Este síndrome se considera grave cuando el docente no es consciente de que lo está padeciendo y comienza a presentar algunos síntomas. Existe una escala de indicios relacionados con este síndrome, cuando el docente sufre por lo menos uno de estos, ya se puede considerar problemático. (Muñoz y Correa, 2012)

Yorulmaz y Altinkurt (2018), en su estudio titulado "El examen del agotamiento de los docentes en Turquía: un meta análisis" utilizaron herramientas estadísticas para identificar las diferencias significativas en diversas variables tales como género, estado civil, asignaturas de enseñanza y entre otras para el diagnóstico del síndrome de burnout en docentes. Una de las conclusiones fue que no existen diferencias significativas en dichas variables, pero identificaron que existe cierta tendencia en la opción género masculino a mayor probabilidad de padecer el síndrome.

Faskhodi Amini y Siyyari (2018), en su artículo titulado: "Dimensiones del compromiso laboral y el agotamiento de los docentes: un estudio de las relaciones entre los docentes de EFL iraníes", estudiaron el modelo de engagement - burnout en maestros teniendo en cuenta que a mayor compromiso manifestaban bajos síntomas del síndrome; asimismo, a más años de docencia o experiencia menor grado de signos de desgaste emocional a diferencia de los docentes que tenían menor compromiso con sus actividades evidenciaban mayor tendencia a desgaste psíquico.

Rodríguez, Guevara y Viramontes (2017), realizaron una investigación que trata el fenómeno del estrés laboral y su manifestación más aguda: el síndrome de burnout. El estudio se desarrolló en docentes de una escuela secundaria mexicana. Los principales resultados presentaron un análisis de la 
problemática de este síndrome a través de cuatro categorías principales: el estrés laboral, el docente, el alumno y el ámbito institucional. En la primera explica la esencia de cómo se percibe el síndrome; en los tres restantes, la percepción y vivencia del problema por el docente y sus actividades; el alumno y lo que le implica convivir con un maestro con este problema y, por último, la institución en función de cómo enfrenta lo que se vive al interior de la misma como consecuencia de la existencia de un docente con estas características.

Rivera, Segarra y Giler (2018), en el estudio realizado sobre el síndrome de burnout en docentes de instituciones de educación superior en la ciudad de Guayaquil tuvo como objetivo describir el síndrome de burnout, midiendo la prevalencia de despersonalización, agotamiento emocional y realización personal, trabajaron bajo la metodología cuantitativa mediante un estudio de campo con una muestra de 159 docentes. Los resultados del estudio arrojaron que el burnout se manifiesta de forma diferente según los años de experiencia en la docencia y de acuerdo a las condiciones del trabajo. Se identificó una relación directa entre el estrés laboral percibido, el agotamiento emocional y los síntomas.

García, Marín y Aguayo (2018), señalaron que los maestros de secundaria son uno de los grupos ocupacionales que presentan los niveles más altos de baja por enfermedad debido al estrés en el lugar de trabajo. Encontraron así que los maestros de secundaria tienen un alto riesgo de síndrome de burnout. Además, existen diferencias estadísticamente significativas entre las pruebas tomadas.

Bedoya, Vega, Severiche y Meza (2017), hicieron una investigación con docentes utilizando el inventario de burnout de Maslach. El objetivo consistió en evaluar el síndrome de quemado (burnout) y factores asociados en docentes de una institución superior del caribe colombiano. Los resultados muestran que la realización personal y los síntomas de estrés se encuentran asociados al género. Los dos fueron más altos en el género femenino. Los docentes que cuentan con solo pregrado y con menor antigüedad reportan mayores puntajes en agotamiento emocional. Se concluye que el agotamiento emocional varía significativamente según área de desempeño de los profesores.

Bulatevych (2017), señala que las manifestaciones del síndrome de burnout en docentes se dan como mecanismos protectores para la defensa emocional de la persona ante situaciones estresantes, teniendo mayor agotamiento emocional los maestros que sufren el síndrome. Asimismo, el entorno laboral contribuye a intensificar o disminuir la incidencia del cansancio psíquico.

Las teorías psicológicas del aprendizaje ofrecen explicaciones sobre el origen del conocimiento y acerca de los procesos lógicos y psicológicos involucrados en el aprendizaje. De esas teorías derivan modelos educativos, el diseño instruccional y estrategias y técnicas pedagógicas que favorecen el aprendizaje, así como estrategias y técnicas evaluativas particulares y con bases científicas. Se aprende mediante ensayo y error o bien observando a otras personas más experimentadas; lo que sigue siendo así en el aprendizaje informal y también en algunas situaciones en la educación formal. (Fonseca y Maria, 2011). Por lo que, en el contexto educativo el burnout es considerado una problemática cuyas consecuencias se ven reflejadas en el desempeño de la función docente y esto su vez repercute en el rendimiento.

En el contexto laboral, el síndrome burnout se manifiesta cuando el individuo presenta estrategias de afrontamiento poco efectivas para enfrentar el estrés provocado no sólo al exceso de la carga laboral sino también inadecuadas relaciones con los colaboradores y 
clientes. En las investigaciones revisadas anteriormente se evidencia que, en el caso de los docentes, el burnout se genera a partir de las exigencias de las diversas funciones que ejercen $\mathrm{y}$ las condiciones de trabajo a las que son expuestos, evidenciándose en la percepción de cansancio, fatiga física, ansiedad, etc.

La trasformación de las sociedades trae consigo el desarrollo y calidad de la educación que se imparte y es importante analizar el papel de las instituciones educativas dentro del proceso de globalización asumiendo un gran protagonismo en la formación de la sociedad, con la responsabilidad de formar personas con un perfil de competencias que garantice la calidad en su aporte a la sociedad en donde se insertarán laboralmente.

En consecuencia, la UNESCO (2015) estable como reto para los docentes: "enfrentar con entusiasmo, dedicación, compromiso, motivación permanente, capacitado y con nivel de todo un profesional los compromisos que la profesión docente implica, y lograr que el reconocimiento social se exprese favorablemente como consecuencia del desempeño del docente de educación básica, ya que la necesidad de que su trabajo sea reconocido lleva implícito un llamado a ésta para que demuestre un compromiso ético y pedagógico con el derecho de todos y cada uno de los estudiantes a una educación pertinente y de calidad.

Una característica del nuevo escenario de la educación, es que es extremadamente demandante, lo cual aumenta la presión y exigencia en los docentes y esto repercute directamente en su vida personal y profesional, pudiendo así afectar seriamente su salud física y mental. Los desafíos para la educación se caracterizan por hacer frente a múltiples cambios que conllevan a transformaciones organizacionales que consisten en asignar nuevas tareas a la actividad docente que van más allá del trabajo en el aula tales como la participación de planes y programas, planeación de actividades docentes y la elaboración de materiales didácticos. Por lo que, la suma de todas estas condiciones puede llegar a convertirse en factores de riesgo que afecten no sólo para los docentes, sino también para los estudiantes que están bajo su resguardo.

Los docentes están inmersos en un contexto social y laboral tan exigente que trae consigo la posibilidad de que el profesional experimente desestabilidad en las diversas áreas de su vida y malestar psicológico. En este sentido existe un deterioro de la salud física y mental que repercute en el compromiso y la satisfacción laboral, por lo que el éxito o el fracaso de una reforma educativa depende de la forma en la cual los docentes interpretan, analizan e intervienen, teniendo como resultado la aceptación o el rechazo de cualquier cambio educativo. (Chávez, y Otros 2014).

La forma en cómo responde el docente a los cambios afecta en su bienestar psicológico en el trabajo, desarrollando procesos ansiosodepresivos como respuesta a un estrés prolongado mejor conocido como Síndrome de Burnout, el cual se presenta en aquellas personas que no fueron capaces de desempeñarse de manera adecuada ante tales cambios y exigencias.

Esta investigación recopila información relevante sobre las condiciones y consecuencias del síndrome de burnout experimentado por docentes para generar posibilidades de atención mediante la creación de espacios donde puedan compartir sus experiencias y ser capacitados para encontrar un equilibrio saludable entre su trabajo y su salud mental.

\section{MÉTODO}

La investigación tuvo un enfoque análisis de la información cualitativo y descriptivo, donde se buscó medir y detallar el síndrome de 
burnout de los docentes en un momento determinado, para lo cual se aplicaron las preguntas del cuestionario validado de Maslach Burnout Inventory (MBI) basado en afirmaciones específicas sobre los tres aspectos del síndrome de burnout con escalas de opinión y de actitudes tipo Likert para su medición. Se aplicaron 59 encuestas a los docentes de una unidad educativa ecuatoriana.

El cuestionario de Maslach Burnout Inventory (MBI) es considerado el instrumento más utilizado en todo el mundo para evaluación de este síndrome. Esta escala tiene una alta consistencia interna y alta fiabilidad, está constituido por 22 ítems en forma de afirmaciones, sobre los sentimientos y actitudes del profesional en su trabajo y hacia los alumnos, su función es medir el desgaste profesional. Este test pretende medir la frecuencia y la intensidad con la que se sufre el Burnout.

Se aplicó la versión traducida y validada de la original que ya ha sido utilizada en numerosos estudios realizados con otro tipo de poblaciones. Se trata de un cuestionario con 7 opciones de respuesta (escala Likert de 0 a 6) que mide los 3 aspectos del síndrome: cansancio emocional, despersonalización y realización personal. Con respecto a las puntaciones se consideran bajas, las que están por debajo de 34, altas puntuaciones en las dos primeras sub escalas y bajas en la tercera que permiten diagnosticar el trastorno.

Subescala de agotamiento emocional. Consta de 9 preguntas, valora la vivencia de estar exhausto emocionalmente por las demandas del trabajo. Puntuación máxima 54

Subescala de despersonalización. Está formada por 5 ítems, valora el grado en que cada uno reconoce actitudes de frialdad y distanciamiento. Puntuación máxima 30

Subescala de realización personal. Se compone de 8 ítems. Evalúa los sentimientos de autoeficacia y realización personal en el trabajo. Puntuación máxima 48.

La clasificación de las afirmaciones dentro del cuestionario fue la siguiente:

- Cansancio emocional: $1,2,3,6,8,13,14,16$, 20.

- Despersonalización: 5, 10, 11, 15, 22.

- Realización personal: 4, 7, 9, 12, 17, 18, 19, 21.

La escala se midió según los siguientes rangos:

$0=$ Nunca

$1=$ Pocas veces al año o menos

2 = Una vez al mes o menos

$3=$ Unas pocas veces al mes o menos

$4=$ Una vez a la semana

$5=$ Pocas veces a la semana

$6=$ Todos los días

Para el cálculo de la puntuación se suman las respuestas dadas a los ítems que se señalan en el cuestionario y los resultados son representados de la siguiente manera:

\begin{tabular}{lll} 
Aspecto evaluado & Preguntas a evaluar & Indicios de Burnout \\
\hline Cansancio emocional & $1-2-3-6-8-13-14-16-20$ & Más de 26 \\
Despersonalización & $5-10-11-15-22$ & Más de 9 \\
Realización personal & $4-7-9-12-17-18-19-21$ & Menos de 34 \\
\hline
\end{tabular}


En la valoración de puntuaciones se toma en cuenta, que: altas puntuaciones en las dos primeras sub escalas y bajas en la tercera definen el síndrome de Burnout. Se analizó de manera detallada los distintos aspectos para determinar el grado del síndrome de burnout de cada docente evaluado, que puede ser más o menos severo dependiendo de si los indicios aparecen en uno, dos o tres ámbitos; y de la mayor o menor diferencia de los resultados con respecto a los valores de referencia que marcan los indicios del síndrome. Este análisis de aspectos e ítems se orientó sobre los puntos fuertes y débiles de cada participante en su labor docente.

\section{RESULTADOS}

Las encuestas permitieron conocer el nivel de burnout en los docentes de acuerdo a su edad, nivel académico, tiempo de trabajo en la institución y en ejercicio de la docencia. La información obtenida de la población de una Unidad Educativa en Ecuador se obtuvo en base a la nómina de docentes que corresponde a 59 docentes. La distribución de la población según el género estuvo constituida en un $70 \%$ por el género femenino, mientras que el género masculino estuvo representado con un $30 \%$.

La población de docentes en la Unidad Educativa ecuatoriana respecto a la edad, dichos resultados se describen en la Figura 1, en donde se destaca que el $46 \%$ de los docentes se encuentran entre los 26 y 35 años, el $42 \%$ de la población está entre los 36 y 45 años, el 7\% representa a los docentes entre 46 y 55 años de edad, el $5 \%$ corresponde a los que tienen mayor de 56 años.

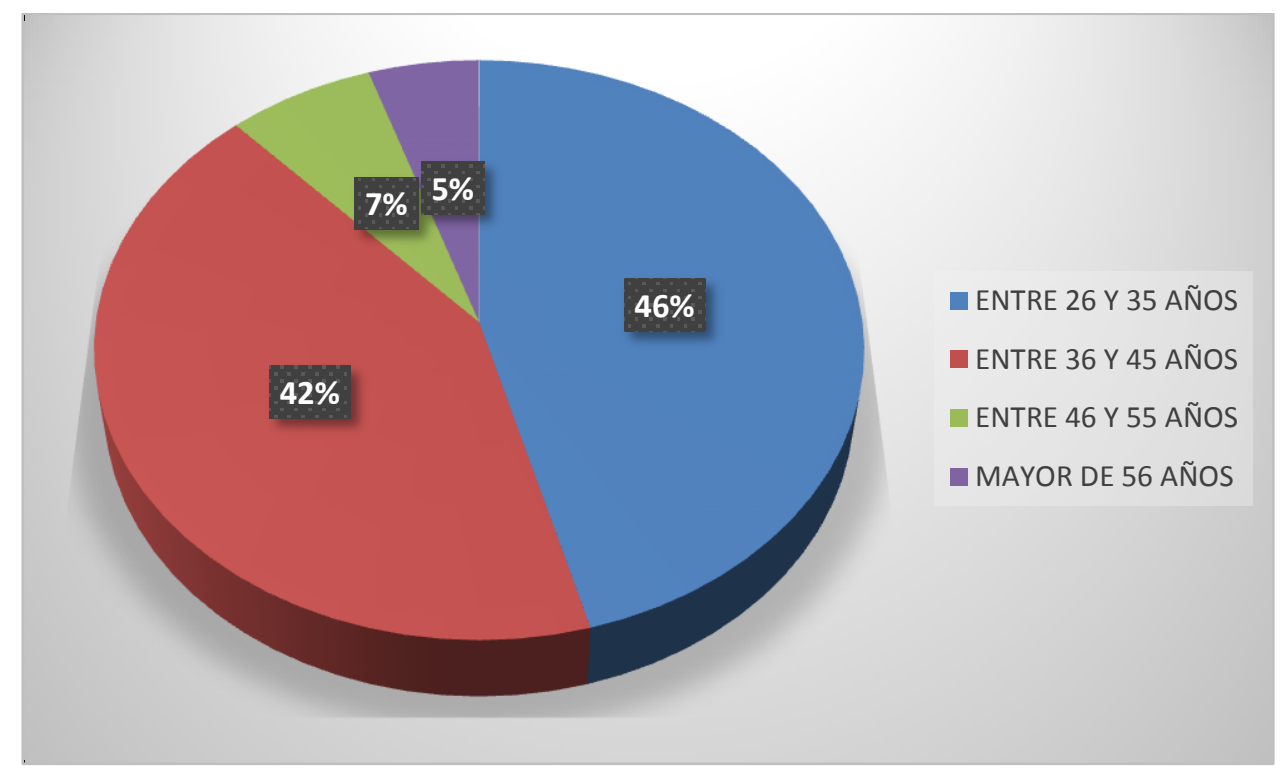

Figura 1. Porcentaje de docentes por edad en la Unidad Educativa Particular Santo Domingo de Guzmán $(\mathrm{N}=59)$ 
En cuanto al nivel de estudios la mayoría de los docentes alcanzan un nivel de especialización con un $36 \%$, el nivel de maestría dentro de la población encuestada fue del $34 \%$, seguido con un $24 \%$ de docentes que tiene un título de pregrado, el $3 \%$ es normalista y el $2 \%$ cuenta con un doctorado.

Respecto al tiempo de antigüedad de los docentes en la institución reflejo que el 56\% indicó trabajar en la institución en un período de 1 a 5 años, el $23 \%$ de docentes indicaron trabajar entre 6 y 10 años, mientras que el 12 $\%$ señaló permanecer en la institución un periodo de 16 a 20 años, el 5\% representa a los docentes que trabajan de 11 a 15 años en la institución, y el 2\% reporto trabajar menos de un año al igual que los docentes que laboran por más de 20 años en la institución educativa en mención.

Aproximadamente el 29\% de los docentes de la unidad educativa analizada padecen de cansancio emocional. Con bajo nivel de burnout se encontraron 42 docentes, con burnout moderado 8, con burnout alto 9 docentes.

Respecto a la despersonalización se puede observar que $17 \%$ de docentes presenta un nivel medio en esta dimensión del burnout. En la tabla 1 se puede apreciar que 43 docentes tienen un nivel bajo de burnout, 10 docentes un burnout medio y 6 docentes tienen un nivel alto de burnout según la dimensión de despersonalización.

Tabla 1. Nivel de despersonalización en los docentes de la Unidad Educativa Particular Santo Domingo de Guzmán (N=59).

Frecuencia

43

10

Válidos

MEDIO

ALTO

6

59

Total
Porcentaje

$73 \%$

$17 \%$

$10 \%$

100,0
Porcentaje acumulado

73

90

100,0
En cuanto a la dimensión de realización personal de los docentes se determinó que el $85 \%$ presenta un alto nivel se realización, $10 \%$ un nivel medio y el $5 \%$ un nivel bajo.

El estudio permitió identificar que muchas de las fuentes de donde deriva el burnout están fuera del sujeto, es decir, en la organización. Sin embargo, la institución estudiada dentro de su planeación estratégica aplica programas dirigidos a la prevención o disminución de esta problemática teniendo como factores principales los entrenamientos en habilidades básicas y avanzadas para enfrentar situaciones que producen estrés. Por esta razón el nivel de realización personal es alto.

Los resultados bajos en los factores: cansancio emocional y despersonalización demuestran la importancia del papel del maestro en el proceso de enseñanzaaprendizaje dentro de la institución. Lo que se observó en el estudio es que la institución procura condiciones aptas y justas para el desarrollo de la labor docente. A nivel organizacional se cuenta con políticas y canales de comunicación pertinentes para prevenir oportunamente el estrés laboral provocado por la falta de información. 
Como se puede apreciar en los resultados, la profesión docente está sujeta a una serie de cambios debido a las demandas de la sociedad por lo que, esta situación ocasiona constantes resistencias. Si se le suman los problemas personales-familiares, se provoca deterioro de la calidad de vida.

El personal docente se enfrenta diariamente a conflictos y situaciones problemáticas relacionadas con la conducta, aulas inadecuadas, ambientes de trabajo, necesidades económicas y burocracia. Esta situación de alguna manera repercute en la salud de los docentes que presentaron un valor alto en cansancio emocional, lo cual ocasiona daños que se manifiestan en síntomas relacionados con su labor diaria. La docencia se considera uno de los trabajos con alto riesgo para desarrollar estrés laboral.

\section{Discusión}

El Síndrome de Burnout ha sido investigado en diferentes profesiones $y$ estudiado desde diversas perspectivas, lo que ha determinado una complejidad en su identificación, método y correlación. Las recientes investigaciones integran la relación del síndrome, no sólo con variables vinculadas con factores organizacionales sino también con variables sociodemográficas y también, se han preocupado por relacionarlo con la forma en que los colaboradores afrontan las situaciones estresantes. Respecto a lo anteriormente expuesto, esta investigación tuvo como objetivo determinar el nivel de burnout en los docentes para lo cual los resultados indicaron que los docentes presentan niveles bajos de síndrome de burnout, una cifra que indica cierta similitud con estudios de la misma naturaleza de ámbito nacional.

Para la recolección de datos se aplicó el cuestionario de MBI (Maslach Burnout Inventory) lo cual generó cierta incertidumbre en los resultados obtenidos ya que entre algunos de los estudios en Latinoamérica sobre el burnout se encuentran validaciones de los instrumentos de Maslach, como por ejemplo para el MBI (Maslach Burnout Inventory), en aras de que la evaluación del burnout sea válida en función del país en que se realiza. El MBI es un cuestionario considerado como "el 'estándar de oro' para evaluar el burnout; sin embargo, ha sido fuertemente criticado a nivel mundial (Díaz y Gómez, 2016).

Por lo tanto, se puede decir que en los países latinoamericanos no se han desarrollado modelos conceptuales del burnout propios del contexto. Esto se hace evidente en el uso de teorías y métodos de medición y evaluación creados en países desarrollados, industrializados, sin la reflexión acerca de la especificidad de los procesos de trabajo-salud dentro de este contexto. Debido a estas observaciones se debería elaborar un instrumento con escalas cualitativas que permita obtener resultados puntuales en futuras investigaciones.

Los datos recopilados en la presente investigación indican que un número reducido de docentes a pesar de no presentar el síndrome, sí muestran niveles medios y altos de las características que lo definen y según estudios similares esto podría afectar en el desarrollo de sus actividades y relaciones con los estudiantes. Por lo tanto, se determina la necesidad de poner atención en el grupo de docentes que se encuentran en esta escala porque se están en riesgo de padecerlo. Esto concuerda con un estudio realizado en docentes de colegios de Cali, Colombia no encontraron niveles severos de burnout en los docentes del colegio público y privado, aunque sí se presentaron algunos indicadores moderados del síndrome en ambas instituciones. En cuanto a las dimensiones del síndrome se determinó que la mayoría de los docentes, tanto del colegio 
público como del privado, presentan niveles normales de Agotamiento Emocional, Despersonalización y Falta de Realización Personal, y un porcentaje bajo presentan niveles moderados de estos. Los indicadores del síndrome de burnout y sus tres dimensiones no presentaron relaciones significativas con el tipo de colegio en el que los docentes trabajan. (Díaz, López, y Varela, 2012).

En el estudio realizado por Rivera, Segarra y Giler (2018) titulado "Síndrome de burnout en docente de instituciones de educación superior" se evidencia que los docentes en instituciones de educación superior en la ciudad de Guayaquil muestran prevalencia baja en las escalas de agotamiento emocional y despersonalización, pero en cuanto a la realización personal se evidencian docentes carentes de respeto, atropellados en sus derechos laborales con baja autoestima, presionados por factores externos a ellos y que su vida probablemente se ha convertido en una rutina. Mientras que en los resultados obtenidos en la investigación en los docentes de la unidad educativa ecuatoriana los niveles de los dos primeros factores y baja, pero se evidencia un nivel alto de realización personal.

En el estudio se pudo evidenciar un alto nivel de realización personal, lo cual muestra una buena respuesta de los docentes logrando desarrollar estrategias de afrontamiento como espacios para la resolución de conflictos, condiciones aceptable en cuanto a la infraestructura, dotación de materiales y herramientas para el desarrollo de su actividad docente y capacidad personal para enfrentar la presión laboral, lo que es contrario a los resultados que se presentaron en el artículo titulado "Prevalencia del burnout en docentes de nivel secundario" publicado en la Revista de Psicología en el año 2019, donde esta dimensión fue la más afectada puesto que se evidenció frustración y desilusión profesional producido en los primeros años de trabajo de los docentes.

Morocho (2019) identificó en su investigación de tesis que la mayor parte de los docentes presentan estrategias de afrontamiento positivas, característica que les permite conllevar y manejar de manera eficaz situaciones problemáticas que causan estrés. Mientras que una pequeña parte del profesorado poseen estrategias negativas, lo que conlleva a un manejo inadecuado de las situaciones estresantes que se les presenta. Estos resultados son similares a los hallazgos en la presente investigación puesto que los docentes de la unidad educativa muestran mantener cierto equilibrio en la carga laboral evitando caer en niveles altos de estrés.

\section{CONCLUSIONES}

La profesión docente es una labor demandante ya que exige el desarrollo de habilidades y competencias intelectuales para la realización eficiente de su trabajo. Además, implica una gran responsabilidad porque se convierten en un modelo a seguir, son formadores de sus estudiantes y su desempeño tiene injerencia en el desarrollo de la sociedad. Los factores mencionados determinan la complejidad del trabajo y esto genera el estrés profesional.

En el análisis de los resultados se evidencia que los docentes evaluados poseen una prevalencia baja del síndrome, aunque existen docentes ubicados en la escala media y una población relativamente pequeña que se encuentra en una prevalencia alta lo que se considera un factor de alerta ya que con facilidad pueden llegar a caer en otros síntomas que complementarían cuadros de burnout.

La realización personal en los docentes es alta, lo cual indica que la mayoría están satisfechos con el trabajo que realizan, 
sintiéndose estancados, sin metas, sin

expectativas personales y laborales.

La determinación de estrategias de afrontamiento con las que cuenta la institución, objeto de estudio cumple con el propósito de evitar y disminuir el estrés laboral, además, para reducir la probabilidad de que se desarrolle el Síndrome de burnout.

En el caso analizado el hecho de otorgar reconocimientos a los altos grados de evaluación docente e incentivar el trabajo, a través de bonos económicos o cantidades extras por desempeño, y comprobantes del mismo se convierten en buenas medidas ante el Burnout.

La generación de un ambiente laboral de buenas relaciones interpersonales, los espacios y momentos de compartir se convierten en situaciones que favorecen el fortalecimiento de los equipos de trabajo, por tanto, debe favorecerse un ambiente de trabajo agradable, compartir emociones $\mathrm{y}$ sentimientos con los compañeros.

A nivel organizacional, es importante eliminar o disminuir los estresores del entorno organizacional que dan lugar al desarrollo del síndrome. Entre los factores más relevantes se encuentra la dedicación al esparcimiento, con jornadas de tiempo libre, donde se disfrute al máximo en actividades placenteras para la persona; y de esta manera se evitará los síntomas del burnout.

En cuanto a la labor docente, la preparación de las clases, la claridad curricular, el manejo de grupo, el espacio dentro del salón y la actualización constante, son elementos que permiten alejarse de sufrir el estrés profesional para evitar el síndrome de burnout se relacionen con otros factores que pueden intervenir en la generación del síndrome.
Bedoya, Elías A, Vega, Nancy E, Severiche, Carlos A, y Meza, María J. (2017). Síndrome de Quemado (Burnout) en Docentes Universitarios: El Caso de un Centro de Estudios del Caribe Colombiano. Formación universitaria, 10(6), 51-58. https://dx.doi.org/10.4067/S071850062017000600006

Bulatevych, N. (2017). Teacher's burnout syndrome: the phenomenology of the process. Polish Journal of Public Health. 127.

https://www.researchgate.net/publicatio n/323189349_Teacher's_burnout_syndro me_the_phenomenology_of_the_process

Chávez Ramírez, D., Pando Moreno, M., Aranda Beltrán, C., y Almeida Perales, C. (2014). Burnout y Work Engagement en Docentes Universitarios de Zacatecas. Ciencia \& trabajo, 16(50), 116-120.

Cruz, D., y Puentes, A. (2017). Relación entre las diferentes dimensiones del síndrome de Burnout $y$ las estrategias de afrontamiento empleadas por los guardas de seguridad de una empresa privada de la ciudad de Tunja. Psicogente,, 268-281.

Díaz, F., \& Gómez, I. (2016). Investigación sobre el síndrome de burnout en latinoamérica entre 2000 y 2010. Psicología desde el Caribe, 121-123.

Díaz, F., López, A., \& Varela, M. (2012). Factores asociados al sindrome de burnout en docentes de colegios de la ciudad de Cali, Colombia. Universitas Psychologica, vol 11, num 1, 217-227.

Faskhodi, AA y Siyyari, M. (2018). Dimensiones del compromiso laboral y el agotamiento de los docentes: un estudio de las relaciones entre los docentes iraníes de inglés como lengua extranjera. Revista Australiana de Educación Docente, 43, 78-93. Obtenido de https://eric.ed.gov/?id=EJ1169149 
Fonseca, H., \& Maria, B. (2011). Teorías del aprendizaje y modelos educativos. $L a$ Revista de Enfermería y Ciencias de la Salud, 71-93.

García Carmona, Marina \& Marín, María \& Aguayo, Raimundo. (2019). Burnout syndrome in secondary school teachers: a systematic review and meta-analysis. Social Psychology of Education. 22. Obtenido de https://www.researchgate.net/publicatio n/328807531_Burnout_syndrome_in_seco ndary_school_teachers_a_systematic_revie w_and_meta-analysis

Green, DE, FH Walkey y AJW Taylor: 1991, "La estructura de tres factores del inventario de agotamiento de Maslach", Journal of Science Behavior and Personality 6, págs. 453-472.

Imbernón, F. (2017). Ser docente en una sociedad compleja: La difícil tarea de enseñar. Barcelona: Grao.

Moreno-Jiménez, Bernardo; Garrosa Hernández, Eva; González Gutiérrez, José Luis (2000). La evaluación del estrés y el burnout del profesorado: el CBP_R. Revista de Psicología del Trabajo y de las Organizaciones-2000. Vol. 16, núm. 2, pp. 151-171.

Obtenido de https://journals.copmadrid.org/jwop/file s/63238.pdf

Morocho Yambay, Jessica Vanessa (2019). Burnout y afrontamiento de los docentes de la unidad educativa "once de noviembre", Riobamba, período octubre 2018 - marzo 2019 (Tesis de pregrado). Universidad Nacional De Chimborazo, Ecuador.

Muñoz, C. (2012). Burnout docente y estrategias de afrontamiento en docentes de primaria y secundaria. Revista Colombiana de Ciencias Sociales, 227-230.

Muñoz, C., \& Correa, M. (15 de 11 de 2012). Burnout docente y estrategias de afrontamiento. Obtenido de file://C:/Users/ANGI/Downloads/DialnetBurnoutDocenteYEstrategiasDeAfrontamie ntoEnDocente-5123799.pdf
Pombo, M. F. et al. (2015). Reforma educativa en ecuador y su influencia en el clima organizacional de los centros escolares. Observatorio de la Econom'ia Latinoamericana, (209).

Rivera, Á; Segarra, J.; Giler, G. (2018) Síndrome de burnout en docentes de instituciones de educación superior. AVFT - Archivos Venezolanos De Farmacología Y Terapéutica. Vol. 37, número 2. Obtenido de file://C:/Users/ANGI/Downloads/15169 -144814482544-1-PB.pdf

Rodríguez Ramírez, J.A.; Guevara Araiza, A.; Viramontes Anaya, E. (2017) Síndrome de burnout en docentes IE Revista de Investigación Educativa de la REDIECH, vol. 8, núm. 14. Red de Investigadores Educativos Chihuahua A. C., México. Obtenido de: http://www.redalyc.org/articulo.oa?id=5 21653267015

Salanova, M. y WB Schaufeli (2000). "Exposición al agotamiento y su relación con el agotamiento", Comportamiento y tecnología de la información, 19, págs. 385-392.

Sánchez-Jiménez B, Flores-Ramos M, Sámano R. Factores relacionados a la presencia del síndrome de burnout en una muestra de enfermeras del sector salud, Ciudad de México. Revista Salud Pública Nutr. 2018;17(3):1-8.

Schaufeli, W. (2005). Burnout en profesores: Una perspectiva social del intercambio. Revista de Psicología del Trabajo y de las Organizaciones., 21(1-2), 15-35. Obtenido de

https://www.wilmarschaufeli.nl/publicati ons/Schaufeli/242.pdf

Schaufeli, WB, Salanova, M., González-romá, V. (2002). La medición del compromiso y el agotamiento: un enfoque analítico de factores confirmatorios de dos muestras. Journal of Happiness Studies 3, 71-92

UNESCO. (2015). Declaración de Incheon. Educación20130: Hacia una educación inclusiva y equitativa de calidad y un aprendizaje a lo largo de la vida para 
todos. Foro Mundial sobre la Educación $2015 . \quad$ Disponible en: http://es.unesco.org/world-educationforum-2015/about-forum/declaracionde-incheon

Yorulmaz, Y.İ. y Altınkurt, Y. (2018). El examen del agotamiento docente en
Turquía: un meta análisis. Revista Turca de Educación, 7, 34-54. Obtenido de https://www.researchgate.net/publicatio n/322772175_The_examination_of_teache r_burnout_in_Turkey_A_meta-analysis 